\title{
Multicentre comparison of self-management in patients with
} COPD

\author{
Ritsuko Wakabayashi ${ }^{1}$, Jean Bourbeau $\mathbb{B}^{2}$, Maria F. Sedeno $\mathbb{B}^{3}$, Takashi Motegi ${ }^{4}$, Tomoko Kutsuzawa ${ }^{5}$, \\ Tetsuya Urano ${ }^{6}$ and Kozui Kida ${ }^{4}$
}

${ }^{1}$ Faculty of Health Care and Nursing, Graduate School of Health Care and Nursing, Juntendo University, Urayasu, Chiba, Japan. ${ }^{2}$ Dept of Medicine, Division of Experimental Medicine, McGill University, McGill University Health Centre, Montreal, QC, Canada. ${ }^{3}$ Research Institute of McGill University Health Centre, Montréal, QC, Canada. ${ }^{4}$ Respiratory Care Clinic Tokyo, Tokyo, Japan. ${ }^{5}$ Faculty of Nursing, Tokai University School of Medicine, Tokai University, Kanagawa, Japan. ${ }^{6}$ School of Medicine, Tokai University, Kanagawa, Japan.

Corresponding author: Ritsuko Wakabayashi (r.wakabayashi@juntendo.ac.jp)

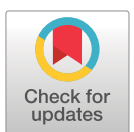

Copyright @The authors 2021

This version is distributed under the terms of the Creative Commons Attribution NonCommercial Licence 4.0. For commercial reproduction rights and permissions contact permissions@ersnet.org

Received: 11 April 2021 Accepted: 21 June 2021

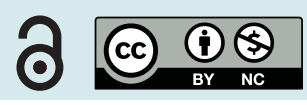

Shareable abstract (@ERSpublications)

This study compared patients' information needs from three institutions, highlighting real-world differences in information needs and the importance of assessing individual needs for self-management in COPD https://bit.ly/3jlPbse

Cite this article as: Wakabayashi R, Bourbeau J, Sedeno MF, et al. Multicentre comparison of self-management in patients with COPD. ERJ Open Res 2021; 7: 00252-2021 [DOI: 10.1183/ 23120541.00252-2021].

\section{Abstract}

In patients with COPD, self-management plays an important role in disease management. Recently, self-management programmes have expanded patient education practices to include a variety of disease management techniques. We hypothesised that COPD patients have insufficient and/or different self-management needs according to institution. We compared information needs of patients between specialised clinics in Canada (SCC) and Japan and a hospital outpatient clinic in Japan (HCJ), all employing different self-management interventions.

This cross-sectional study evaluated patients' information needs for disease management using the Lung Information Needs Questionnaire (LINQ). Furthermore, we assessed pulmonary function tests, modified Medical Research Council (mMRC) dyspnoea scale and frequencies of hospitalisations and emergency visits.

The total number of patients was 183. Those attending SCC were younger ( $p=0.047$ ), with lower forced expiratory volume in $1 \mathrm{~s} \%$ predicted $(\mathrm{p}<0.0001)$, and scored higher on the mMRC dyspnoea scale. Total LINQ scores showed differences between institutions $(\mathrm{p}<0.0001)$. There was no difference for the smoking domain; however, SCC recorded significantly lower information needs for all other domains $(\mathrm{p}<0.02)$. No significant difference in emergency visits was seen between institutions, but HCJ recorded the highest rate of emergency visits, while SCC had significantly higher rates of hospitalisation $(p=0.004)$. Differences were seen for frequency and duration of education between institutions.

These results highlight the differences in information needs by institution and the importance of assessing individual needs. We believe, despite representing only one aspect of self-management, our findings reflect real-world circumstances, adding to the argument that self-management education should be structured, but flexible, to meet the changing needs of COPD patients.

\section{Introduction}

COPD, characterised by airflow obstruction [1], is increasing in prevalence worldwide and is now the third leading cause of mortality [2]. Patients with poorly managed COPD require frequent hospitalisation, and the rates of mortality due to exacerbations have been reported to be approximately $11-24 \%$ [3]. Therefore, self-management is considered an integral component of the chronic care model of disease management [4].

Self-management programmes for COPD patients are primarily aimed at teaching the skills needed to control the disease and correct unhealthy behaviours to improve wellbeing [5]. Informing patients on how to avoid disease complications, such as exacerbations, has been thought to be the key to successful disease 
management. However, recent literature has reported that traditional methods of self-management with the target of preventing exacerbations alone are outdated [6]. Instead, patients should be encouraged to acquire and apply self-management skills to enhance healthier behaviours in their daily lives.

Furthermore, self-management programmes can vary widely by institution. Strategies can range in time and scope from simple didactic instruction to more multifaceted self-management interventions. BouRBEAU et al. [7] reported that a comprehensive self-management education can provide information and skills that emphasise disease control though behaviour modification, thus increasing self-efficacy with the goal of improving clinical outcomes. Recently, self-management programmes have expanded their patient education practices to include a variety of disease management interventions such as smoking cessation, medication, exercise and nutrition, which is seen as critically important $[6,8]$.

We reported previously that an integrated education for COPD patients evaluated by the Lung Information Needs Questionnaire (LINQ) [9] improved patients' information needs, reduced disease symptoms and hospitalisations [3]. The LINQ was able to evaluate the knowledge that patients obtained through self-management programmes and could pinpoint the individual needs of the patient. For the present study, we hypothesise that patients with COPD might receive insufficient or different information for self-management programmes between facilities. The aim of this study is to compare information needs of patients with COPD between two specialised respiratory clinics and a general hospital outpatient clinic.

\section{Materials and methods}

\section{Patient selection}

This cross-sectional prospective observational multicentre study included patients across three institutions from Canada and Japan. Patients were recruited from the following institutions: 1) the Montreal Chest Institute of the McGill University Health Centre (specialised clinic, Canada (SCC)), Montreal, Canada, a specialised respiratory clinic with a well-established self-management education programme for patients with COPD; 2) the Respiratory Care Clinic (specialised clinic, Japan (SCJ)), Nippon Medical School, Tokyo, Japan, another specialised COPD clinic with a continuous education programme based on LINQ domains for the self-management of COPD; and 3) Tokai University Hospital (general hospital clinic, Japan (HCJ)), Kanagawa, Japan, a general hospital where patients visited the outpatient clinic for consultation regarding COPD, but without a standard or tailored self-management programme.

Inclusion criteria were as follows: patients aged $>40$ years with a formal diagnosis of COPD; a post-bronchodilator forced expiratory volume in $1 \mathrm{~s}\left(\mathrm{FEV}_{1}\right)$ of $<80 \%$ predicted; $\mathrm{FEV}_{1}$ /forced vital capacity (FVC) $<0.7$; those who attended regularly scheduled appointments for $\geqslant 6$ months at their primary institution; and a smoking history of $\geqslant 10$ pack-years. Patients were excluded if they had a history of atopy or any apparent asthmatic features, a diagnosis of dementia or were illiterate in English and/or French (patients in Canada) or Japanese (patients in Japan).

All patients included in this study provided written informed consent and were able to withdraw at any time. This study, registered at Tokai University, was approved by the ethics committees of all participating institutions (approval number 12R-049).

\section{Outcome assessments}

The LINQ was used to assess patients' knowledge of their disease at each setting. The LINQ is a self-administered questionnaire that measures the information needs of patients with COPD. Briefly, the LINQ is divided into six domains, and each domain is scored as follows: an understanding of COPD (0$4)$; the use of medications ( $0-5)$; the avoidance of exacerbations (0-6); risks of smoking (0-3); exercise $(0-5)$; and nutrition (0-2). The sum of these scores is the total LINQ score (0-25). A higher score indicates a higher need for information on disease self-management. The total LINQ score provides an overview of the patient's information needs, and the individual domain scores identify specific needs.

\section{Outcome measurements}

Pulmonary function parameters, including $\mathrm{FEV}_{1}$, vital capacity and FVC, were measured according to the guidelines of the American Thoracic Society (ATS)/European Respiratory Society (ERS) [10] using equipment for lung function testing. The predicted value was calculated based on reference values from the ATS/ERS [10] (patients in Canada) and the Japanese Respiratory Society (JRS) [11] (patients in Japan).

COPD severity was assessed by spirometric classifications based on the ATS/ERS statement [8]. 
The severity of dyspnoea was evaluated using the modified Medical Research Council (mMRC) dyspnoea scale [12].

Exacerbations were self-reported from the previous year at the initial consultation for each setting. Exacerbations were defined as an increase in severity of the following respiratory symptoms: dyspnoea; cough and sputum volume; and sputum purulence that led to a change in medication or treatment, such as antibiotics or systematic corticosteroids, or admission to hospital [1]. Emergency visits and hospitalisations for the previous 12 months were self-reported by participants at initial interview.

\section{Self-management programmes by institution}

Specialised clinic, Canada

SCC utilised the Living Well with COPD (LWWCOPD) self-management programme (www. livingwellwithcopd.com). Briefly, the LWWCOPD is an evidence-based self-management programme which is used extensively throughout Canada and other countries. The LWWCOPD was delivered by one 30-min education session per week, for 7-8 weeks, either one-to-one or in a group setting. An extensive range of teaching materials are available for health professionals and patients, and the education sessions range from basic information on COPD to integration of healthy behaviours and self-management strategies. LWWCOPD includes an action plan for acute exacerbation that is customised for each patient, including a contact list and a symptom-monitoring list for different situations.

\section{Specialised clinic, Japan}

Each patient received an individually tailored programme. Treatment and healthcare management plans were created for each patient's self-management needs, including an action plan for exacerbations. Education was delivered via monthly individual sessions with $\geqslant 30 \mathrm{~min}$ spent with each patient. All patients were provided with an education booklet based on the six domains of LINQ [13] which was used during each session. An action plan including instructions for exacerbation was provided to each patient. Education was conducted in an interactive style, with patients encouraged to ask questions about current or past sessions.

\section{General hospital clinic, Japan}

Patients visited the outpatient clinic for consultation once or twice a month regarding their disease symptoms and medications. During individual consultations, patients were asked about self-management techniques they may have been concerned about. Nurses instructed patients on self-management techniques for $\geqslant 15$ min based on patient inquires using third-party publicly accessible materials based on guidelines from the JRS [14]. Pulmonologists and nurses, at their own discretion, further prepared self-management materials for patients building on past consultation visits or when they believed there was a lack of knowledge. Advice, but no formal action plan, was used during consultations.

\section{Statistical analysis}

We calculated the mean \pm SD with categorical variables expressed as percentages. Comparison among multiple groups were assessed by ANOVA and Chi-squared test or Fisher's exact tests (for categorical variables). A p-value $<0.05$ was considered significant. Data were analysed using Statistical Package for the Social Sciences, version 25.0 for Windows (IBM SPSS, Chicago, IL, USA).

Results

Patient characteristics for each institution are shown in table 1. The total number of patients was 183 (SCC $\mathrm{n}=45$, SCJ $\mathrm{n}=105$, HCJ n=33); 28 were female. SCC had the youngest patient population (SCC 69.0 years, SCJ 72.6 years, HCJ 72.9 years) ( $\mathrm{p}=0.047$ ). Furthermore, SCC had lower $\mathrm{FEV}_{1}$ values (SCC $35.3 \%$ pred, SCJ $68.9 \%$ pred, HCJ $60.1 \%$ pred $)(\mathrm{p}<0.0001)$ and scored higher on the mMRC dyspnoea scale (SCC 2.87, SCJ 1.01, HCJ 1.39) $(\mathrm{p}<0.0001)$.

COPD severity of patients is shown in figure 1 . For the SCC, $20 \%$ of patients had moderate COPD, while $40 \%$ had each of severe and very severe COPD. There were no mild COPD patients at SCC. In contrast, at the SCJ, mild and moderate COPD patients comprised $71.4 \%$, while severe COPD patients comprised $25.7 \%$ of the cohort and $3 \%$ of patients were very severe. For the HCJ, mild and moderate COPD patients made up $75.8 \%$ of the total, while $12.1 \%$ were severe and very severe COPD (four patients each).

The mean total LINQ scores showed significant differences between institutions $(\mathrm{p}<0.0001)$ (SCC 3.91, SCJ 6.29, HCJ 9.79) (table 2). Although there were no differences in the smoking domain between groups, SCC recorded lower information needs for disease knowledge $(\mathrm{p}=0.013)$, medications $(\mathrm{p}<0.0001)$, avoidance of exacerbations $(\mathrm{p}<0.0001)$, exercise $(\mathrm{p}=0.007)$ and nutrition $(\mathrm{p}=0.014)$ (table 2$)$. When 


\section{TABLE 1 Patient characteristics}

\begin{tabular}{lcccc} 
& $\begin{array}{c}\text { Specialised clinic, } \\
\text { Canada }\end{array}$ & $\begin{array}{c}\text { Specialised clinic, } \\
\text { Japan }\end{array}$ & $\begin{array}{c}\text { General hospital clinic, } \\
\text { Japan }\end{array}$ & p-value \\
\hline Patients & 45 & 105 & 33 & \\
\hline Age (years) & $68.96 \pm 9.02$ & $72.56 \pm 7.75$ & $72.88 \pm 5.89$ & 0.0474 \\
\hline Male/female & $29 / 16$ & $98 / 7$ & $28 / 5$ & $<0.0001$ \\
\hline FVC (L) & $2.16 \pm 0.77$ & $3.18 \pm 0.70$ & $2.79 \pm 0.79$ & $<0.0001$ \\
\hline FEV (L) & $0.95 \pm 0.42$ & $1.67 \pm 0.65$ & $2.06 \pm 3.28$ & $<0.0001$ \\
\hline FEV 1 \% pred & $35.31 \pm 14.64$ & $68.88 \pm 25.23$ & $60.14 \pm 22.32$ & $<0.0001$ \\
\hline mMRC dyspnoea scale $^{\text {d }}$ & $2.87 \pm 0.81$ & $1.01 \pm 0.96$ & $1.39 \pm 0.83$ & $<0.0001$ \\
\hline
\end{tabular}

Data are presented as $\mathrm{n}$ or mean $\pm \mathrm{SD}$, unless otherwise stated. FVC: forced vital capacity; $\mathrm{FEV}_{1}$ : forced expiratory volume in $1 \mathrm{~s}$; mMRC: modified Medical Research Council.

comparing institutions in Japan, SCJ patients revealed lower information needs than HCJ patients for disease knowledge, avoidance of exacerbations and exercise domains.

There was no significant difference in emergency visits for the previous year among institutions, but the HCJ recorded the highest rate of emergency visits. SCC had a significantly higher number of hospitalisations for the previous year when compared with Japanese institutions ( $p=0.0004)$ (table 3).

The self-management education settings for each institution are shown in table 4. There were differences seen between medical systems and facilities between Canada and Japan. Whereas patients in Canada were treated by their primary care physicians, Japanese patients were free to choose between physicians and hospitals. All settings in this study were outpatient clinics, but educators and self-management interventions varied. The SCC included respiratory physicians, nurses, physiotherapists, respiratory therapists and a dietician, while the SCJ was staffed by respiratory physicians, nurses and a dietician. The HCJ included only respiratory physicians and nurses. Furthermore, there were differences in duration for education and frequency of visits between institutions. Patients attending the SCC spent 30 min per visit once a week, and those attending the SCJ spent 30 min per visit once a month, while those attending the HCJ were allotted 15 min per education session, once or twice a month.

\section{Discussion}

This study compared the information needs of patients assessed using the LINQ from two specialised respiratory clinics and a general hospital outpatient clinic and found that self-management of COPD differed by institution. Those attending specialised COPD settings had a better understanding of their

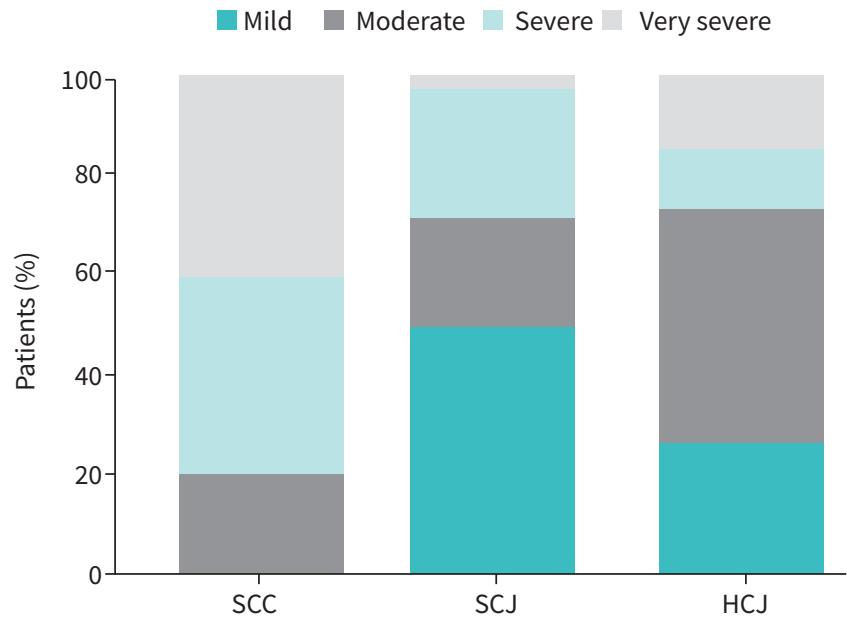

FIGURE 1 Severity of COPD in patients by institution. Patients attending the specialised clinic, Canada (SCC) were more severe than patients from the specialised clinic, Japan (SCJ) and the general hospital clinic, Japan $(\mathrm{HCJ})(\mathrm{p}<0.0001)$. 


\begin{tabular}{|c|c|c|c|c|}
\hline & $\begin{array}{l}\text { Specialised } \\
\text { clinic, Canada }\end{array}$ & $\begin{array}{l}\text { Specialised } \\
\text { clinic, Japan }\end{array}$ & $\begin{array}{l}\text { General hospital } \\
\text { clinic, Japan }\end{array}$ & p-value \\
\hline Patients & 45 & 105 & 33 & \\
\hline \multicolumn{5}{|l|}{ LINQ domain } \\
\hline Disease knowledge (range 0-4) & $0.86 \pm 0.84$ & $1.12 \pm 0.66$ & $1.60 \pm 1.20$ & 0.0126 \\
\hline Medications (range $0-5$ ) & $0.27 \pm 0.54$ & $0.48 \pm 0.81$ & $0.79 \pm 1.34$ & $<0.0001$ \\
\hline Avoidance of exacerbations (range $0-6$ ) & $0.98 \pm 1.14$ & $2.47 \pm 1.60$ & $4.52 \pm 1.52$ & $<0.0001$ \\
\hline Smoking cessation (range $0-3$ ) & $0.16 \pm 0.37$ & $0.04 \pm 0.19$ & $0.12 \pm 0.42$ & 0.091 \\
\hline Exercise (range 0-5) & $1.02 \pm 0.94$ & $1.18 \pm 0.84$ & $1.91 \pm 1.35$ & 0.0070 \\
\hline Nutrition (range $0-2$ ) & $0.62 \pm 0.83$ & $1.00 \pm 0.39$ & $0.85 \pm 0.76$ & 0.0138 \\
\hline LINQ total score (range $0-25$ ) & $3.91 \pm 2.63$ & $6.29 \pm 2.63$ & $9.79 \pm 3.67$ & $<0.0001$ \\
\hline
\end{tabular}

disease and were better able to respond to exacerbations compared to those attending the general hospital. Although the specialised clinics in this study shared similarities in their structured self-management programmes and comprehensive approach, the SCC scored better on the LINQ, despite the SCJ programme being based on the LINQ. We believe this might be due to differences in disease severity that could have affected patient outcomes.

\section{Information needs for self-management}

Insufficient information in the self-management of COPD can lead to poor clinical outcomes. In this study, information needs were assessed using the LINQ, which has been proven to highlight areas of insufficient knowledge for self-management in COPD patients [3]. The LINQ, which is a self-administered, 16-item questionnaire has been shown to be effective at detecting the information needs of individuals and might have greater benefits for nonspecialised institutions without integrated self-management programmes. Since information needs and the number of emergency visits were highest for the HCJ compared with the specialised clinics, the LINQ could be considered a good starting point to assess the knowledge of each patient.

Self-management programmes used by specialised clinics provide comprehensive information to patients, but for general clinics without self-management programmes, it can be difficult to provide self-management information due to time and resource limitations. This was the case in the present study where the HCJ recorded higher information needs for all LINQ domains, except smoking cessation. It has been reported that patients who consulted their general practitioners were dissatisfied with the treatment options available to them [15], namely that they were limited to medicine use and smoking cessation rather that pulmonary rehabilitation or other aspects of self-management. In our study, LINQ domain scores were low for the smoking and medication domains for all settings, despite a significant difference seen for the medication domain. We consider that a reason for this could be that COPD patients might have enough information for smoking and medications, since these self-management components are now widely implemented as standard interventions in most medical settings.

Therefore, using LINQ in nonspecialised settings to objectively assess various gaps in self-management information for COPD might contribute to more efficient and individualised self-management interventions or assist in the decision to refer patients to more specialised COPD settings.

TABLE 3 Number of emergency visits and hospitalisations by institution

\begin{tabular}{lcccc} 
& $\begin{array}{c}\text { Specialised clinic, } \\
\text { Canada }\end{array}$ & $\begin{array}{c}\text { Specialised clinic, } \\
\text { Japan }\end{array}$ & $\begin{array}{c}\text { General hospital clinic, } \\
\text { Japan }\end{array}$ & p-value \\
\hline Patients & 45 & 105 & 33 & \\
ER visits per year & $0.53 \pm 0.81$ & $0.47 \pm 0.83$ & $0.91 \pm 1.63$ & 0.3331 \\
\hline Hospitalisations per year & $1.07 \pm 1.89$ & $0.07 \pm 0.35$ & $0.67 \pm 1.37$ & 0.0004 \\
\hline Data are presented as n or mean \pm SD, unless otherwise stated. ER: emergency room. &
\end{tabular}




\section{TABLE 4 Comparison of self-management settings}

\begin{tabular}{|c|c|c|c|}
\hline & Specialised clinic, Canada & $\begin{array}{l}\text { Specialised clinic, } \\
\text { Japan }\end{array}$ & $\begin{array}{l}\text { General hospital clinic, } \\
\text { Japan }\end{array}$ \\
\hline Consultation & Referral & Free access & Free access \\
\hline Setting & Clinic, outpatient & Clinic, outpatient & Hospital, clinic, outpatient \\
\hline Educators & $\begin{array}{c}\text { Physician, nurse, physiotherapist, respiratory } \\
\text { therapist, dietician }\end{array}$ & $\begin{array}{l}\text { Physician, nurse, } \\
\text { dietician }\end{array}$ & Physician, nurse \\
\hline Pulmonary rehabilitation & Yes & No & No \\
\hline Education programme & LWWCOPD & LINQ & No \\
\hline Action plan & Yes & Yes & No \\
\hline Education & Every visit & Every visit & When deemed necessary \\
\hline Duration & $30 \mathrm{~min}$ & $30 \mathrm{~min}$ & $15 \mathrm{~min}$ \\
\hline
\end{tabular}

\section{Disease severity and hospitalisation rates}

Exacerbations of COPD develop from an acute worsening of a patient's condition from a stable state which results in a change in medications or hospitalisations. It has been reported that the number of hospitalisations per year is positively correlated to the degree of COPD severity [16], which was similar to the present study. Despite having lower information needs, the SCC patients had significantly higher rates of hospitalisation and dyspnoea, which is probably due to higher disease severity.

The present study revealed that patients at Japanese institutions were older, predominantly male, and had less severe COPD and lower mMRC dyspnoea scale scores. Since this study was observational in design, patients were not controlled for age or disease severity. However, these findings are similar to a literature review by IsHII et al. [17], which reported that patients from Japan had less severe COPD, less dyspnoea and reported lower exacerbation rates than patients from other countries. Although there was no definitive reason as to why these findings are constantly reported, it is considered that extrinsic factors such as lifestyle or better access to healthcare may have contributed to these results.

\section{Integrated self-management}

The American College of Chest Physicians and Canadian Thoracic Society guideline on the prevention of acute exacerbations of COPD states that specially trained staff for education and case management is needed to supervise educational interventions [18].

In our study, we noted a difference in medical staff between specialised clinics and the general hospital outpatient clinic. There were overlaps seen for medical workers at each specialised clinic which employed multidisciplinary staff members, whereas the general hospital was staffed with only physicians and nurses. Although this study included patients who received consultations for $\geqslant 6$ months prior to the evaluation by LINQ, it did not investigate the total duration or quality of education patients received. Therefore, the absence of a multidisciplinary medical team may have negatively affected the HCJ patients' knowledge on a variety of disease management techniques.

However, it is ultimately the decision of each institution to identify optimal care for their patients according to the resources of each institution. For the SCC, as patients had more severe COPD, their comprehensive approach to self-management, including pulmonary rehabilitation, may have contributed to better adherence and retained education than for the Japanese settings. For the SCJ, since patients had predominantly stable COPD, less integrated care may be more beneficial.

\section{Limitations}

There were several limitations to this study. Although we compared self-management in patients across three facilities, the sample size, gender and age of patients differed greatly between settings. However, this is in line with the aforementioned review in which patients in Japan are older and predominantly male. Moreover, disease severity for the SCC was much higher than for the Japanese population. This might be explained by the difference in the referral system used in Canadian settings whereby stable patients may have been treated longer by their primary care physicians rather than independently seeking out specialised care, although further study is needed to confirm these differences. 
Another limitation could be the underlying factors for exacerbations. We did not assess comorbidities or body mass index in this study and these factors may have contributed to the higher hospitalisation rate for the SCC. Furthermore, we found that frequency of visits and the time self-management education was delivered varied widely between settings, especially between specialised and nonspecialised clinics. Many studies have tried to identify the most suitable self-management programme; however, self-management with an emphasis on individual patient's needs over the course of their disease might be considered the most beneficial to the patient, regardless of age, gender or disease severity.

Furthermore, motivation may have been a factor in patients who visited specialised clinics to those visiting the general hospital. Although motivation was not assessed in this study, there has been growing interest in the assessment of motivation in self-management of COPD. Therefore, future studies are needed to assess the outcomes in motivation for self-management.

\section{Conclusion}

This study showed that patients' information needs for the self-management of COPD differed by institution, and that a formal, individualised self-management education was beneficial in retaining or enhancing the patient's self-management information, and in the avoidance of exacerbations. We believe that our findings, although only one aspect of self-management, reflect real-world circumstances adding to the argument that self-management education should be structured, but flexible, to meet the changing needs of COPD patients.

Provenance: Submitted article, peer reviewed.

Conflict of interest: None declared.

Support statement: This study was supported by Grant-in-Aid for Scientific Research JP23890212. Funding information for this article has been deposited with the Crossref Funder Registry.

\section{References}

1 Global Initiative for Chronic Obstructive Lung Disease. Global Strategy for Diagnosis, Management, and Prevention of Chronic Obstructive Pulmonary Disease 2021 Report. www.goldcopd.org/. Date last updated: 4 January 2021. Date last accessed: 4 January 2021.

2 World Health Organization. The Top 10 Causes of Death. www.who.int/news-room/fact-sheets/detail/ the-top-10-causes-of-death Date last updated: 4 January 2021. Date last accessed: 4 January 2021.

3 Wakabayashi R, Motegi T, Yamada K, et al. Efficient integrated education for older patients with chronic obstructive pulmonary disease using the Lung Information Needs Questionnaire. Geriatr Gerontol Int 2011; 11: 422-430.

4 Adams SG, Smith PK, Allan PF, et al. Systematic review of the chronic care model in chronic obstructive pulmonary disease prevention and management. Arch Intern Med 2007; 167: 551-561.

5 Effing TW, Bourbeau J, Vercoulen J, et al. Self-management programmes for COPD: moving forward. Chron Respir Dis 2012; 9: 27-35.

6 Effing TW, Vercoulen JH, Bourbeau J, et al. Definition of a COPD self-management intervention: International Expert Group consensus. Eur Respir J 2016; 48: 46-54.

7 Bourbeau J, Julien M, Maltais F, et al. Reduction of hospital utilization in patients with chronic obstructive pulmonary disease: a disease-specific self-management intervention. Arch Intern Med 2003; 163: 585-591.

8 Celli BR, Decramer M, Wedzicha JA, et al. An Official American Thoracic Society/European Respiratory Society Statement: research questions in chronic obstructive pulmonary disease. Am J Respir Crit Care Med 2015; 191: e4-e27.

9 Hyland ME, Jones RC, Hanney KE. The Lung Information Needs Questionnaire: development, preliminary validation and findings. Respir Med 2006; 100: 1807-1816.

10 Miller MR, Hankinson J, Brusasco V, et al. Standardisation of spirometry. Eur Respir J 2005; 26: 319-338.

11 Japanese Respiratory Society. The predicted values of spirometry and arterial blood gas analysis in Japanese. J Jpn Resp Soc 2001; 39: S1e-S17.

12 Mahler DA, Wells CK. Evaluation of clinical methods for rating dyspnea. Chest 1988; 93: 580-586.

13 Kida K, Yoshioka H, Yamaguchi K, et al. Comprehensive Respiratory Care Using LINQ - Patient Education to Enhance Self-management Ability. Tokyo, Igaku-Shoin, 2006; pp. 30-97.

14 Japanese Respiratory Society. Guideline for Diagnosis and Management of COPD (Chronic Obstructive Pulmonary Disease). Therapy and Management. 4th Edn. Tokyo, Japanese Respiratory Society, 2013.

15 Molin KR, Langberg H, Lange P, et al. Disease self-management in patients with moderate COPD: a thematic analysis. Eur Clin Respir J 2020; 7: 1762376. 
Tsoumakidou M, Tzanakis N, Voulgaraki O, et al. Is there any correlation between the ATS, BTS, ERS and GOLD COPD's severity scales and the frequency of hospital admissions? Respir Med 2004; 98: 178-183.

17 Ishii T, Nishimura M, Akimoto A, et al. Understanding low COPD exacerbation rates in Japan: a review and comparison with other countries. Int J Chron Obstruct Pulmon Dis 2018; 13: 3459-3471.

18 Criner GJ, Bourbeau J, Diekemper RL, et al. Prevention of acute exacerbations of COPD: American College of Chest Physicians and Canadian Thoracic Society Guideline. Chest 2015; 147: 894-942. 